\title{
Direct Oral Anticoagulants after Ischemic Stroke: Which Patient? Which Drug? And How Early?
}

\author{
Gian Marco De Marchis ${ }^{1}{ }^{\circ}$ \\ ${ }^{1}$ Department of Neurology, University Hospital and University of \\ Basel, Basel, Switzerland \\ Hämostaseologie 2021;41:31-34.
}

\begin{abstract}
Address for correspondence Gian Marco De Marchis, MD, MSc, Department of Neurology/Stroke Center, University Hospital Basel, Petersgraben 4, 4031 Basel, Switzerland (e-mail: gian.demarchis@usb.ch).
\end{abstract}

\begin{abstract}
Keywords

- stroke

- atrial fibrillation

- anticoagulation

- timing

Direct oral anticoagulants (DOACs) are recommended over vitamin $\mathrm{K}$ antagonists (VKAs) in patients with atrial fibrillation (AF) and ischemic stroke. The main advantage of DOAC over VKA is the lower rate of bleeding and mortality. This review covers challenges clinicians can encounter when treating patients with AF and ischemic stroke, including timing of DOAC start and ongoing randomized clinical trials, appropriate dosing, and available comparative evidence across DOACs. For patients without AF but with an ischemic stroke, the review outlines the role of DOACs. Finally, the risk of thrombotic events associated with specific DOAC reversal agents and DOAC pausing is reviewed.
\end{abstract}

\section{Introduction}

Atrial fibrillation (AF) increases the risk of ischemic stroke up to five times, and is associated with more severe and twice as fatal strokes as compared with other etiologies. ${ }^{1,2}$ For patients with an ischemic stroke linked to $\mathrm{AF}$, current guidelines recommend direct oral anticoagulants (DOACs) over vitamin K antagonists (VKAs), except for AF in the presence of a mechanical heart valve and moderate-to-severe mitral stenosis. ${ }^{3,4}$ The recommendation is based on four pivotal, phase-3 randomized clinical trials (RCTs) comparing DOACs to VKAs in patients with AF: ARISTOTLE (apixaban), RE-LY (dabigatran), ENGAGE AF-TIMI 48 (edoxaban), and ROCKET $\mathrm{AF}$ (rivaroxaban). ${ }^{5-8}$ The main advantage of DOACs over VKAs was the halved rate of intracranial hemorrhage (ICH)resulting in a lower mortality-with a similar rate of stroke and systemic embolism (SE). In this brief review, we discuss the role of DOACs among patients with ischemic stroke.

\section{Appropriate Direct Oral Anticoagulant Dosing}

RE-LY and ENGAGE AF were the only RCTs that randomized AF patients to two different DOAC doses-in RE-LY: dabigatran $150 \mathrm{mg}$, $110 \mathrm{mg}$ twice daily; in ENGAGE AF: edoxaban
$60 \mathrm{mg}, 30 \mathrm{mg}$ daily. ${ }^{6,7}$ The allocation to the low-dose trial arm was randomized and independent of renal function. In a prespecified meta-analysis, the low dabigatran and edoxaban doses were associated with an increased risk of ischemic stroke compared with VKAs (relative risk: 1.28 , 95\% confidence interval [CI]: $1.02-1.60 ; p=0.045)$, and-like the full doses-with less bleedings $(0.65,0.43-1.00 ; p=0.05){ }^{9} \mathrm{~A}$ retrospective study confirmed that reduced DOAC doses are associated with an increased risk of ischemic stroke. ${ }^{10}$ Since all DOACs are eliminated mainly through the kidney, renal impairment mandates a reduction of the DOAC dose. In the pivotal RCTs-with the exception of RE-LY6 -patients with renal impairment received a reduced DOAC dose in ARISTOTLE (apixaban $2.5 \mathrm{mg}$ [instead of $5 \mathrm{mg}$ ] twice daily in $4.7 \%$ of the overall cohort), ${ }^{5}$ ROCKET-AF (rivaroxaban $15 \mathrm{mg}$ [instead of $20 \mathrm{mg}$ ] daily in $21 \%$ ), ${ }^{8}$ and ENGAGE-AF (edoxaban $30 \mathrm{mg}$ [instead of $60 \mathrm{mg}$ ] or $15 \mathrm{mg}$ [instead of $30 \mathrm{mg}$ ] daily in $25 \%){ }^{7}$ In the ORBIT-AF registry with 5,738 patients, 1 in 8 patients received DOAC doses inconsistent with labeling: $9.4 \%$ were underdosed and $3.4 \%$ overdosed. Underdosing was associated with a $26 \%$ higher risk of cardiovascular hospitalization and overdosing with an almost twofold risk of all-

\section{received}

November 8, 2020

accepted after revision

December 3, 2020 (c) 2021. Thieme. All rights reserved. Georg Thieme Verlag KG,

Rüdigerstraße 14,

70469 Stuttgart, Germany
DOI https://doi.org/

10.1055/a-1329-2523. ISSN 0720-9355. 
cause mortality. ${ }^{11}$ This underscores not to reduce the DOAC dose, unless mandated by the label. Fear of bleeding alone is not an indication to reduce the DOAC dose.

\section{DOAC versus VKA after an Ischemic Stroke}

Can we generalize one-to-one the findings of the RCT to patients with AF and ischemic stroke? No. In the four pivotal RCTs, the majority of patients with AF did not have a history of ischemic stroke, transient ischemic attack (TIA), or SE (19\% in ARISTOTLE, 20\% in RE-LY, 28\% in ENGAGE-AF, and 55\% in ROCKET-AF). In a meta-analysis on 20,500 patients with AF and previous stroke or TIA, DOACs were associated with a significant reduction of recurrent stroke/SE (relative risk reduction: $13.7 \%$, absolute risk reduction: $0.78 \%$, number needed to treat to prevent one event: 127) and ICH (relative risk reduction: $46.1 \%$, absolute risk reduction: $0.88 \%$, number needed to treat: 113 ) over 1.8 to 2.8 years. ${ }^{12}$ Recently, two multicenter observational studies compared DOACs to VKAs among patients with AF and ischemic stroke or TIA. The first study was an individual-patient data meta-analysis with 4,912 patients from Europe and Japan, with a median follow-up of 1.2 years. In the DOAC group, less ICH occurred (hazard ratio [HR]: $0.42 ; 95 \% \mathrm{Cl}$ : 0.24-0.71) with no significant differences in the rate of recurrent ischemic stroke (HR: 0.91; 95\% CI: 0.70-1.19) and all-cause mortality (HR: 0.83; $95 \%$ CI: $0.68-1.03)$. The second study included 1,251 patients with AF and stroke from the United States. ${ }^{13}$ Follow-up was shorter (90 days), and the median time between strokes to therapeutic international normalized ratio was 8 days (interquartile range: $4-13$ ). In contrast with the prior study, there were fewer recurrent ischemic strokes in the DOAC group (HR: 0.51; 95\% CI: 0.29-0.87), whereas the risk of ICH did not differ compared with the VKA group (HR: 0.57; 95\% CI: 0.22-1.48). The different conclusions suggest that the benefit of DOAC over VKA evolves over time. In the first few weeks after stroke, immediate anticoagulation with DOAC translates into fewer ischemic strokes, compared with the delayed treatment effect with VKA. On the longer run, the more selective the anticoagulation achieved with DOACs, the lower the ICH rate. If an ICH occurs, its 30-day fatality is lower among patients treated with DOACs than those treated with VKAs, as seen in a recent meta-analysis of 11 observational studies (relative risk: $0.70,95 \% \mathrm{CI}: 0.51-0.95){ }^{14}$

\section{How Early?}

A further reason why RCTs cannot be generalized to patients with ischemic stroke is the timing of DOAC start after an ischemic stroke. In all four RCTs, a key exclusion criterion was ischemic stroke within 1 week and 6 months prior to randomization, with a median time between ischemic stroke and randomization over 1 year. ${ }^{15,16}$ The reason for such long waiting times was the fear of hemorrhagic transformation. The "1-3-6-12" rule of thumb stems from the pre-DOAC era and is not backed by evidence. The rule advocates to start oral anticoagulation (OAC) after 1 day in TIA, 3 days in minor strokes, 6 days in moderate strokes, and 12 days in large infarcts. ${ }^{17}$ However, in clinical practice, DOACs tend to be started much earlier compared to RCTs: median timing of DOAC initiation was between 5 and 11 days in the four observational studies with clinical follow-up of at least 3 months. ${ }^{18-21}$ Despite such early starts, the fear of hemorrhagic transformation of the infarcted brain did not materialize. Rather, the risk of recurrent ischemic stroke was three times higher than the risk of ICH (annualized average rate of 6.6 vs. $2.2 \%$ for recurrent stroke and $\mathrm{ICH}$, respectively). ${ }^{22}$ This evidence is observational and randomized trials omparing early versus late starts are currently ongoing-their results are eagerly awaited (ELAN [Switzerland/International], NCT03148457; TIMING [Sweden], NCT02961348; OPTIMAS [United Kingdom], EduraCT 2018-003859-38; START [United States], NCT03021928). The best current practice is to enroll patients in these trials, rather than start DOAC early based on the available nonrandomized evidence.

\section{Comparative DOAC Efficacy and Safety in Atrial Fibrillation}

We cannot state that one DOAC is better than the other, since there are no randomized controlled trials comparing DOACs head to head. Moreover, the baseline risk of strokemeasured with the $\mathrm{CHADS}_{2}$ score-varied considerably across the four RCTs (mean of 3.5 in ROCKET-AF, 2.8 in ENGAGE-AF, and 2.1 in RE-LY and ARISTOTLE), making it difficult to compare DOACs across trials. ${ }^{5-8}$ There are, however, observational studies that compared DOACs to each other. One of the latest studies included 52,476 patients with AF from nationwide registries in Norway and compared dabigatran, rivaroxaban, and apixaban over a median follow-up of 18 months. ${ }^{23}$ Overall, the risk of stroke or SE was similar across the DOACs in the propensity score analysis, while the risk of major bleeding was significantly lower for dabigatran and apixaban compared with rivaroxaban. Of interest, in the subgroup with a history of stroke (13\% of the overall cohort), rivaroxaban was superior to both apixaban and dabigatran in preventing recurrent strokes, with a similar bleeding rate.

The largest comparative study was a retrospective analysis on 434,046 patients from the Medicare and Medicaid in the United States (the ARISTOPHANES study). ${ }^{24}$ Apixaban was associated with a lower rate of stroke and major bleeds compared with dabigatran and rivaroxaban (stroke -28 and $-20 \%$, respectively; bleedings: -22 and $-45 \%$, respectively). Dabigatran, compared with rivaroxaban, had a similar rate of stroke with a lower bleeding rate $(-29 \%)$. In the subgroup with a history of stroke (12\% of the overall cohort), the advantage of apixaban over rivaroxaban and dabigatran in protecting against stroke/SE dissipated, with rivaroxaban still being associated with a higher bleeding rate compared with apixaban and dabigatran. The median follow-up of 4 months in ARISTOPHANES is a major limitation, given that DOACs for AF are prescribed long term. The conflicting results in secondary prevention-with rivaroxaban favored in one study, disfavored in the other-highlight the limitations of nonrandomized studies. Such studies should be rather seen as hypothesis-generating, rather than change practice. 


\section{DOACs in Absence of AF in Patients with Ischemic Stroke}

Among patients without AF-but with coronary heart disease, peripheral artery disease, or carotid stenosis $>50 \%-$ rivaroxaban $2.5 \mathrm{mg}$ twice daily combined with aspirin $100 \mathrm{mg}$ daily was compared with aspirin $100 \mathrm{mg}$ alone in the COMPASS trial among 27,395 patients. ${ }^{25}$ Patients with a stroke $\geq 1$ month earlier were allowed in the trial, where they represented $3.8 \%$ of patients. The primary outcome-a composite of cardiovascular death, stroke, or myocardial infarction-occurred in fewer patients in the rivaroxaban-plusaspirin group than in the aspirin-alone group (HR: 0.76, 95\% CI: $0.66-0.86, p<0.001) .{ }^{25}$ The benefit in the combination group was driven by the reduction in ischemic stroke events over aspirin alone (0.9 vs. $1.6 \% /$ year $){ }^{26}$ The types of ischemic stroke that were reduced most were cardioembolic and embolic strokes of unknown origin, suggesting a modest but relevant anticoagulatory effect of rivaroxaban $2.5 \mathrm{mg}$ twice daily. ${ }^{27}$ Major bleedings occurred more often in the rivaroxaban-plus-aspirin group (HR: 1.70, 95\% CI: 1.40-2.05, $p>0.001$ ), with no significant difference in intracranial or fatal bleeding. ${ }^{25}$ Among patients with a history of stroke, the risk of major hemorrhage was higher (HR: 3.79, 95\% CI: 1.07-13.4, $p=0.04$ ), calling for caution when prescribing the combination therapy after a stroke. ${ }^{26}$ Following an ischemic stroke, the waiting time should be at least 1 month prior to start of the dual antithrombotic therapy.

There is no indication for DOACs among patients with an ischemic stroke with an embolic pattern on brain magnetic resonance imaging but without any identified cause (embolic stroke of unknown source): compared with aspirin, rivaroxaban and dabigatran brought no benefit in the NAVIGATE and RESPECT-ESUS trials, respectively. ${ }^{28,29}$

\section{Specific Reversal Agents and Thrombotic Risk}

Idarucizumab is a monoclonal antibody fragment that binds dabigatran. In RE-VERSE AD, idarucizumab reversed the anticoagulant effect of dabigatran within 4 hours among 503 patients with uncontrolled bleeding or about to undergo an urgent procedure. ${ }^{30}$ Andexanet alfa is a modified recombinant form of human factor Xa. In ANNEXA-4, andexanet alfa reduced antifactor Xa activity due to factor Xa inhibitors among 352 patients with acute major bleeding (ICH in two-thirds of patients). ${ }^{31}$ Neither study had a control arm. In patients requiring idarucizumab or andexanet alfa, the incidence of thrombotic events was 5.5\% (95\% CI: $2.0-10.1 \%$ ) within 30 to 90 days, and allcause mortality was $13.3 \%$ (95\% CI: 9.6-17.5\%), according to a recent meta-analysis of 13 studies on idarucizumab and three studies on andexanet alfa. ${ }^{32}$ Most thrombotic events were stroke and venous thromboembolism. Compared with idarucizumab, more thrombotic events were seen with andexanet alfa (3.3 vs. 10.6\%). Firm comparative conclusions are not possible, given the low number of studies with andexanet alfa.

Thrombotic events do not seem to be directly related to the antidotes, since most thrombotic events occurred when resumption of DOAC was delayed or never occurred.
In ANNEXA-4, no thrombotic event occurred after restart of oral anticoagulation. ${ }^{31}$ Therefore, and despite the absence of solid efficacy, reversal of DOACs with specific agents should be considered in patients with moderate to severe bleeding that cannot be stopped by other means (such as in intracranial bleeding), and where the bleeding does not already seem to be fatal. After a major bleeding, it appears prudent to resume $\mathrm{OAC}$ as soon as the clinician judges it to be safely possible.

\section{Conflict of Interest}

Dr. De Marchis reports personal fees and other from Bayer, personal fees from BMS/Pfizer, outside the submitted work.

\section{References}

1 Wolf PA, Abbott RD, Kannel WB. Atrial fibrillation as an independent risk factor for stroke: the Framingham Study. Stroke 1991;22 (08):983-988

2 Lin HJ, Wolf PA, Kelly-Hayes M, et al. Stroke severity in atrial fibrillation. The Framingham Study. Stroke 1996;27(10): $1760-1764$

3 Klijn CJ, Paciaroni M, Berge E, et al. Antithrombotic treatment for secondary prevention of stroke and other thromboembolic events in patients with stroke or transient ischemic attack and non-valvular atrial fibrillation: a European Stroke Organisation guideline. Eur Stroke J 2019;4(03):198-223

4 January CT, Wann LS, Calkins H, et al;Writing Group Members. 2019 AHA/ACC/HRS focused update of the 2014 AHA/ACC/HRS guideline for the management of patients with atrial fibrillation: a report of the American College of Cardiology/American Heart Association Task Force on Clinical Practice Guidelines and the Heart Rhythm Society. Heart Rhythm 2019;16(08): e66-e93

5 Granger CB, Alexander JH, McMurray JJ, et al;ARISTOTLE Committees and Investigators. Apixaban versus warfarin in patients with atrial fibrillation. N Engl J Med 2011;365(11):981-992

6 Connolly SJ, Ezekowitz MD, Yusuf S, et al;RE-LY Steering Committee and Investigators. Dabigatran versus warfarin in patients with atrial fibrillation. N Engl J Med 2009;361(12):1139-1151

7 Giugliano RP, Ruff CT, Braunwald E, et al;ENGAGE AF-TIMI 48 Investigators. Edoxaban versus warfarin in patients with atrial fibrillation. N Engl J Med 2013;369(22):2093-2104

8 Patel MR, Mahaffey KW, Garg J, et al;ROCKET AF Investigators. Rivaroxaban versus warfarin in nonvalvular atrial fibrillation. $\mathrm{N}$ Engl J Med 2011;365(10):883-891

9 Ruff CT, Giugliano RP, Braunwald E, et al. Comparison of the efficacy and safety of new oral anticoagulants with warfarin in patients with atrial fibrillation: a meta-analysis of randomised trials. Lancet 2014;383(9921):955-962

10 Paciaroni M, Agnelli G, Caso V, et al. Causes and risk factors of cerebral ischemic events in patients with atrial fibrillation treated with non-vitamin $\mathrm{K}$ antagonist oral anticoagulants for stroke prevention. Stroke 2019;50(08):2168-2174

11 Steinberg BA, Shrader P, Thomas L, et al;ORBIT-AF Investigators and Patients. Off-label dosing of non-vitamin $\mathrm{K}$ antagonist oral anticoagulants and adverse outcomes: the ORBIT-AF II Registry. J Am Coll Cardiol 2016;68(24):2597-2604

12 Ntaios G, Papavasileiou V, Diener HC, Makaritsis K, Michel P. Nonvitamin-K-antagonist oral anticoagulants versus warfarin in patients with atrial fibrillation and previous stroke or transient ischemic attack: an updated systematic review and meta-analysis of randomized controlled trials. Int J Stroke 2017;12(06): 589-596 
13 Yaghi S, Mistry E, Liberman AL, et al. Anticoagulation type and early recurrence in cardioembolic stroke: the IAC study. Stroke 2020;51(09):2724-2732

14 Tsivgoulis G, Katsanos AH, Seiffge DJ, et al;RAF, RAF-DOAC, CROMIS-2, SAMURAI, NOACISP, Erlangen, Verona Registry Collaborators. Fatal intracranial haemorrhage occurring after oral anticoagulant treatment initiation for secondary stroke prevention in patients with atrial fibrillation. Eur J Neurol 2020;27(08): 1612-1617

15 Hankey GJ, Patel MR, Stevens SR, et al;ROCKET AF Steering Committee Investigators. Rivaroxaban compared with warfarin in patients with atrial fibrillation and previous stroke or transient ischaemic attack: a subgroup analysis of ROCKET AF. Lancet Neurol 2012;11(04):315-322

16 Diener HC, Connolly SJ, Ezekowitz MD, et al;RE-LY study group. Dabigatran compared with warfarin in patients with atrial fibrillation and previous transient ischaemic attack or stroke: a subgroup analysis of the RE-LY trial. Lancet Neurol 2010;9(12): $1157-1163$

17 Steffel J, Verhamme P, Potpara TS, et al;ESC Scientific Document Group. The 2018 European Heart Rhythm Association Practical Guide on the use of non-vitamin K antagonist oral anticoagulants in patients with atrial fibrillation. Eur Heart J 2018;39(16): $1330-1393$

18 Seiffge DJ, Traenka C, Polymeris A, et al. Early start of DOAC after ischemic stroke: risk of intracranial hemorrhage and recurrent events. Neurology 2016;87(18):1856-1862

19 Mizoguchi T, Tanaka K, Toyoda K, et al;SAMURAI Study Investigators. Early initiation of direct oral anticoagulants after onset of stroke and short- and long-term outcomes of patients with nonvalvular atrial fibrillation. Stroke 2020;51(03):883-891

20 Paciaroni M, Agnelli G, Falocci N, et al. Early recurrence and major bleeding in patients with acute ischemic stroke and atrial fibrillation treated with non-vitamin-K oral anticoagulants (RAFNOACs) study. J Am Heart Assoc 2017;6(12):e007034

21 Wilson D, Ambler G, Banerjee G, et al;Clinical relevance of Microbleeds in Stroke (CROMIS-2) collaborators. Early versus late anticoagulation for ischaemic stroke associated with atrial fibril- lation: multicentre cohort study. J Neurol Neurosurg Psychiatry 2019;90(03):320-325

22 Seiffge DJ, Werring DJ, Paciaroni M, et al. Timing of anticoagulation after recent ischaemic stroke in patients with atrial fibrillation. Lancet Neurol 2019;18(01):117-126

23 Rutherford OW, Jonasson C, Ghanima W, Söderdahl F, Halvorsen S. Comparison of dabigatran, rivaroxaban, and apixaban for effectiveness and safety in atrial fibrillation: a nationwide cohort study. Eur Heart J Cardiovasc Pharmacother 2020;6(02):75-85

24 Lip GYH, Keshishian A, Li X, et al. Effectiveness and safety of oral anticoagulants among nonvalvular atrial fibrillation patients. Stroke 2018;49(12):2933-2944

25 Eikelboom JW, Connolly SJ, Bosch J, et al;COMPASS Investigators. Rivaroxaban with or without aspirin in stable cardiovascular disease. N Engl J Med 2017;377(14):1319-1330

26 Sharma M, Hart RG, Connolly SJ, et al. Stroke outcomes in the COMPASS trial. Circulation 2019;139(09):1134-1145

27 Perera KS, Ng KKH, Nayar S, et al. Association between low-dose rivaroxaban with or without aspirin and ischemic stroke subtypes: a secondary analysis of the COMPASS trial. JAMA Neurol 2020;77(01):43-48

28 Hart RG, Sharma M, Mundl H, et al;NAVIGATE ESUS Investigators. Rivaroxaban for stroke prevention after embolic stroke of undetermined source. N Engl J Med 2018;378(23):2191-2201

29 Diener HC, Sacco RL, Easton JD, et al;RE-SPECT ESUS Steering Committee and Investigators. Dabigatran for prevention of stroke after embolic stroke of undetermined source. N Engl J Med 2019; 380(20):1906-1917

30 Pollack CV Jr, Reilly PA, van Ryn J, et al. Idarucizumab for dabigatran reversal - full cohort analysis. N Engl J Med 2017; 377(05):431-441

31 Connolly SJ, Crowther M, Eikelboom JW, et al;ANNEXA-4 Investigators. Full study report of andexanet alfa for bleeding associated with factor Xa inhibitors. N Engl J Med 2019;380(14):1326-1335

32 Rodrigues AO, David C, Ferreira JJ, Pinto FJ, Costa J, Caldeira D. The incidence of thrombotic events with idarucizumab and andexanet alfa: a systematic review and meta-analysis. Thromb Res 2020;196:291-296 